\title{
Fast Food Ingestion for Lunch Delays Postprandial Lipid Metabolism in Young Women
}

\author{
Maiko Kato ${ }^{1}$, Akihiro Yoshida ${ }^{2}$, Michitaka Naito ${ }^{1,}$, \\ ${ }^{1}$ Division of Nutrition \& Health, School \& Graduate School of Life Studies, Sugiyama Jogakuen University, Nagoya, Japan \\ ${ }^{2}$ Department of Clinical Laboratory, Nakatsugawa Municipal General Hospital, Nakatsugawa, Japan
}

\section{Email address:}

naito@sugiyama-u.ac.jp (M. Naito)

${ }^{*}$ Corresponding author

\section{To cite this article:}

Maiko Kato, Akihiro Yoshida, Michitaka Naito. Fast Food Ingestion for Lunch Delays Postprandial Lipid Metabolism in Young Women. Journal of Food and Nutrition Sciences. Vol. 5, No. 3, 2017, pp. 116-121. doi: 10.11648/j.jfns.20170503.18

Received: March 30, 2017; Accepted: April 14, 2017; Published: May 22, 2017

\begin{abstract}
We investigated the effect of fast food on diurnal lipid and carbohydrate profiles in healthy young Japanese women. Eight healthy young women with the apolipoprotein E3/3 phenotype were studied on two occasions in a randomized crossover design. On one occasion, the subjects consumed common foods at breakfast (9:00), lunch (13:00), and dinner (19:00) (C trial). On the other occasion, they consumed common foods at breakfast and dinner, but they consumed fast food (a cheeseburger, fried potatoes, and cola) at lunch (F trial). Blood samples were taken at 9:00, 11:00, 13:00, 15:00, and 19:00, and at 9:00 the next morning. In the $C$ trial, the serum triglyceride (TG) concentration at 19:00 was significantly lower compared with that at 13:00, but in the F trial, it was significantly higher at 15:00, 17:00, and 19:00 compared with that at 13:00. The incremental area under the curve for TG ( $\triangle \mathrm{AUC}-\mathrm{TG})$ (13:00 to 9:00 the next morning) in the $\mathrm{F}$ trial was significantly larger than that in the $\mathrm{C}$ trial. The concentration of fasting remnant lipoprotein-cholesterol (RemL-C) the next morning was positively correlated with $\triangle \mathrm{AUC}$-TG (13:00 to 9:00 the next morning). In conclusion, compared with the conventional diet, the intake of fast food at lunch delayed the postprandial TG metabolism and the serum TG concentration did not return to baseline (before lunch) even before dinner in healthy young Japanese women.
\end{abstract}

Keywords: Postprandial Lipidemia, Triglyceride-Rich Lipoprotein, Triglyceride, Remnant

\section{Introduction}

Fast food refers to food that can be prepared and served quickly by a take-out restaurant. Fast food is filling, usually cheap, quite convenient, and moreover tastes good, so it is popular, particularly among young people. However, the food is often made with high-fat meat, refined grains, added sugar, and fats, and is usually high in calories, fat, cholesterol, and sodium. Accordingly, eating too much fast food can lead to serious health problems such as obesity, metabolic syndrome, hyperlipidemia, diabetes mellitus, hypertension, cardiovascular disease, and cancer.

Zilversmit [1] first proposed that postprandial lipoprotein metabolism may be one of the major causes of atherosclerosis, and since then several studies have shown that postprandial triglyceride (TG) level is an independent predictor of cardiovascular disease, even when adjusting for fasting TG and high-density lipoprotein-cholesterol (HDL-C) levels [2, 3]. In contemporary life, particularly in Japan and western countries, the true 'fasting' period is confined to only 2-3 $\mathrm{h}$ per day, and most of the day is really spent in a 'postprandial' state [4].

Fast food items such as hamburgers usually contain high amounts of fat and are consumed with beverages such as cola and milk shakes that are sweetened with high fructose syrup or sucrose. We have previously shown that the ingestion of a fructose-containing beverage combined with high-fat food caused a delay in the clearance of exogenous (i.e., intestinal) and endogenous (i.e., hepatic) TG-rich lipoproteins and exacerbated postprandial lipidemia even in young healthy women [5-7].

In Japan, it has been reported that high school and university students tend to eat fast food such as hamburgers, French fries, and fried chicken particularly for lunch [8]. Therefore, in the present study, we compared the effect of 
consuming fast food (hamburgers, fried potatoes, and cola) and a common, non-fast-food diet for lunch on diurnal lipid and carbohydrate profiles in young healthy Japanese women. The combination of a hamburger, fried potato, and cola is a representative model of fast food lunch in Japanese youth.

\section{Methods}

\subsection{Subjects}

Eight young healthy Japanese women with normal body mass index (BMI) $\left(18.5 \leq-<25 \mathrm{~kg} / \mathrm{m}^{2}\right)$, normal ovarian cycles, and apolipoprotein $\mathrm{E}$ (apoE) phenotype $3 / 3$ were enrolled as participants. All of the subjects were non-smokers, had no apparent acute or chronic illnesses, and were not taking any medicines or dietary supplements. This study was approved by the Ethical Committee of Sugiyama Jogakuen University School of Life Studies in accord with the Helsinki Declaration, and each subject provided written informed consent for study participation.

\subsection{Anthropometric and Body Composition Measurement}

Body weight and height were measured according to standard methods. Waist circumference was assessed as the abdominal girth at the level of the umbilicus, hip circumference was measured at the level of the greater trochanters, and the waist-to-hip $(\mathrm{W} / \mathrm{H})$ ratio was calculated. Body composition, including the visceral fat area (VFA), was analyzed by an eight-polar bioelectrical impedance method using InBody720 (Biospace, Tokyo, Japan).

\subsection{Experimental Design}

Each subject was studied on two occasions. At each session, the subjects took 3 meals, i.e., breakfast, lunch, and dinner. On one occasion, the subjects ingested fast food (hamburger, fried potatoes, and cola) for lunch ( $\mathrm{F}$ trial), and on the other occasion, they ingested a common, non-fast-food diet for lunch ( $\mathrm{C}$ trial). The two sessions were performed in a randomized crossover design. There was an interval of 4 weeks between the sessions to minimize the confounding effects of menstrual cycle on the metabolism.

The breakfast and dinner ingested in the $\mathrm{C}$ and $\mathrm{F}$ trials were made up of the same common diet. Subjects ingested breakfast at 9:00 after an overnight fast, lunch at 13:00, and dinner at 19:00 on the experimental day.

Venous blood samples were drawn from the antecubital vein before breakfast (9:00), at 11:00, before lunch (13:00), at 15:00, before dinner (19:00), and the next morning (9:00). Blood samples were obtained in a supine position. During the session, the subjects avoided exercise and eating except for the 3 meals, but had free access to water.

\subsection{Test Foods}

The nutritional composition of the test foods is shown in Table 1. No other intake except water was allowed during the study day. Prepared frozen food (Nichirei, Tokyo, Japan) was used except for the lunch in the F trial. The fast food ingested for lunch included a cheeseburger (approx. $120 \mathrm{~g}$, McDonald's, Tokyo, Japan), fried potatoes (medium size, approx. $135 \mathrm{~g}$, McDonald's), and cola (medium-size Coca-Cola, $350 \mathrm{~mL}$, Coca-Cola Journey, Tokyo, Japan). The cola contained $16.8 \mathrm{~g}$ fructose, $12.6 \mathrm{~g}$ glucose, and $8.1 \mathrm{~g}$ sucrose.

Table 1. Composition of trial foods.

\begin{tabular}{llllll}
\hline C trial & & & & \\
\hline & & Breakfast & Lunch & Dinner & Total \\
\hline Energy & $(\mathrm{kcal})$ & 519 & 529 & 574 & 1622 \\
Carbohydrate & $(\mathrm{g})$ & 72.5 & 77.4 & 81.0 & 231 \\
Protein & $(\mathrm{g})$ & 20.7 & 23.9 & 23.4 & 68 \\
Fat & $(\mathrm{g})$ & 15.1 & 12.2 & 16.2 & 43 \\
Salt & $(\mathrm{g})$ & 2.3 & 1.9 & 2.0 & 6.2 \\
\hline & & & & & \\
\hline F trial & & & & & \\
\hline & & Breakfast & Lunch & Dinner & Total \\
\hline Energy & $(\mathrm{kcal})$ & 528 & 878 & 577 & 1983 \\
Carbohydrate & $(\mathrm{g})$ & 71.8 & 129.7 & 85.4 & 287 \\
Protein & $(\mathrm{g})$ & 21.1 & 20.8 & 16.8 & 59 \\
Fat & $(\mathrm{g})$ & 16.0 & 32.9 & 16.7 & 66 \\
Salt & $(\mathrm{g})$ & 2.1 & 2.5 & 2.7 & 7.3 \\
\hline
\end{tabular}

\subsection{Biochemical Analysis}

The serum samples were immediately refrigerated at $4^{\circ} \mathrm{C}$ or frozen at $-80^{\circ} \mathrm{C}$ until analysis. The concentration of glucose (Glu) was measured using the mutarotase-glucose oxidase method (Wako, Osaka, Japan). The concentration of insulin was measured using a chemiluminescent enzyme immunoassay (Fujirebio, Tokyo, Japan). Insulin resistance was evaluated according to the homeostasis model assessment for insulin resistance (HOMA-IR) [9]. The hemoglobin A1c (HbAlc) level was measured using the latex agglutination method (Fujirebio) and expressed as the National Glycohemoglobin Standardization Program (NGSP) value. The concentration of free fatty acids (FFA) (Eiken Chemical, Tokyo, Japan) was measured enzymatically. The concentration of total cholesterol (TC) was measured enzymatically (Sysmex, Hyogo, Japan). The concentration of HDL-C was measured using the direct method (Fujirebio), while the concentration of low-density lipoprotein-cholesterol (LDL-C) was calculated using the Friedewald formula. The concentration of TG was measured enzymatically (Sekisui Medical, Tokyo, Japan).

The TG concentration in the TG-rich lipoproteins was estimated using a homogeneous assay (VLDL-TGTM, Shino-test, Tokyo, Japan) [10], and shown as hepatic triglyceride-rich lipoprotein-triglyceride (hTRL-TG) [6]. The concentration of remnant-like particle-triglyceride (RLP-TG) was measured using an immunosorbent assay (Otsuka Pharmaceutical, Tokyo, Japan), and the concentration of remnant lipoprotein-cholesterol (RemL-C) using a homogeneous assay (MetaboRead ${ }^{\mathrm{TM}}$, Kyowa Medex, Tokyo, Japan) [11].

The concentration of apolipoproteins (apo) AI, AII, B(100), CII, CIII, and E were measured using an immunoturbidimetric method (Sekisui Medical). The concentration of apoB48 was measured by chemiluminescent enzyme immunoassay 
(Fujirebio). The apoE phenotype was measured using the isometric electrophoresis method (Phenotyping ApoE IEF System $^{\mathrm{TM}}$, Joko, Tokyo, Japan).

\subsection{Chronological Quantification of Lipid and Glucose Metabolism}

The chronological changes in the concentrations of TG and Glu were calculated as the difference from the baseline mean value (considered to be 0 at 13:00), and shown as $\Delta \mathrm{TG}$ and $\triangle \mathrm{Glu}$, respectively, and were quantified by calculating the incremental area under the curve ( $\triangle \mathrm{AUC})$, which was defined as the difference between the area under the curve and the area below the baseline (13:00) concentration from 13:00 to 19:00, or from 13:00 to 9:00 the next morning.

\subsection{Statistics}

All data were expressed as mean \pm SEM. The statistical analyses were performed using SPSS ver. 19 (IBM, Tokyo, Japan). Normal distribution of data was verified using the Shapiro-Wilk test. Non-normally distributed raw data (insulin and apoE) were distributed normally when logarithmically transformed, and analyzed using parametric statistics. Differences in the chronological course compared with baseline were analyzed using the paired $t$-test with the Bonferroni correction. The differences in the values at each time point between the trials were analyzed using the paired $t$-test. Correlation between $\triangle A U C-T G(13: 00-9: 00)$ and fasting serum TG, RLP-TG, hTRL-TG, RemL-C, apoB48, Glu, and insulin in the next morning was analyzed using Pearson's correlation coefficient. $p<0.05$ was considered significant in all analyses.

\section{Results}

The physical characteristics and fasting blood chemistry data are shown in Table 2 and the fasting blood chemical data in Table 3. There were no significant differences in any of the physical and fasting blood chemical data between the two trials. The nutritional intake on the day before the trial was not different between the trials, and was similar to the values for young Japanese women in the National Nutritional Survey [12] (data not shown). The physique of the subjects was also average for young Japanese women because their height and BMI were similar to the values reported in the survey.

The diurnal change in serum TG concentration is shown in Figure 1. In Figure 2A, changes in the TG levels are shown in terms of the difference from baseline (13:00) before lunch. In the $\mathrm{C}$ trial, $\triangle \mathrm{TG}$ was not different from baseline at 15:00 or 17:00, and decreased compared to baseline at 19:00. However, in the $\mathrm{F}$ trial, $\Delta \mathrm{TG}$ increased at 15:00 and 17:00, and did not return to baseline even at 19:00 before dinner. $\triangle \mathrm{AUC}-\mathrm{TG}$ (13:00-9:00) was significantly larger in the $\mathrm{F}$ trial compared to the $\mathrm{C}$ trial (Figure $2 \mathrm{~B}$ ).

In Table 4, the correlations between $\triangle \mathrm{AUC}-\mathrm{TG}$ from 13:00 to 9:00 the next morning and the fasting concentrations of TG,
RLP-TG, hTRL-TG, RemL-C, apoB48, Glu, and insulin are shown. $\triangle A U C-T G$ was significantly correlated with RemL-C and hTRL-TG in the $\mathrm{C}$ trial, and RLP-TG and RemL-C in the $\mathrm{F}$ trial. $\triangle \mathrm{AUC}-\mathrm{TG}$ also tended to be correlated with hTRL-TG in the $\mathrm{F}$ trials $(p=0.076)$.

The diurnal change of the serum Glu concentration is shown in Fig. 3. No differences from the baseline at 9:00 before breakfast were observed. There was no difference between the trials.

Table 2. Physical characteristics of the subjects.

\begin{tabular}{lllll}
\hline Age & $($ year $)$ & 21.4 & \pm & 0.3 \\
Height & $(\mathrm{cm})$ & 158.6 & \pm & 1.7 \\
Waist & $(\mathrm{cm})$ & 71.6 & \pm & 2.4 \\
Hip & $(\mathrm{cm})$ & 88.3 & \pm & 2.0 \\
W/H & & 0.81 & \pm & 0.02 \\
Weight & $(\mathrm{kg})$ & 51.7 & \pm & 2.3 \\
BMI & $\left(\mathrm{kg} / \mathrm{m}^{2}\right)$ & 20.6 & \pm & 0.9 \\
\% Body fat & $(\%)$ & 27.9 & \pm & 2.1 \\
Skeletal muscle mass & $(\mathrm{kg})$ & 19.8 & \pm & 0.6 \\
VFA & $\left(\mathrm{cm}^{2}\right)$ & 30.6 & \pm & 5.2 \\
\hline
\end{tabular}

Table 3. Clinical characteristics of the trials.

\begin{tabular}{lllllllll}
\hline & & \multicolumn{3}{c}{ C trial } & \multicolumn{3}{c}{ F trial } & $p$ value \\
\hline Glucose & $(\mathrm{mg} / \mathrm{dL})$ & 83.7 & \pm & 1.0 & 85.3 & \pm & 2.7 & $\mathrm{~ns}$ \\
Insulin & $(\mu \mathrm{IU} / \mathrm{mL})$ & 6.1 & \pm & 0.3 & 7.4 & \pm & 1.5 & $\mathrm{~ns}$ \\
HbA1c & $(\%)$ & 5.2 & \pm & 0.1 & 5.1 & \pm & 0.1 & $\mathrm{~ns}$ \\
HOMA-IR & & 1.3 & \pm & 0.1 & 1.6 & \pm & 0.3 & $\mathrm{~ns}$ \\
FFA & $(\mu \mathrm{Eq} / \mathrm{L})$ & 448.9 & \pm & 52.2 & 363.4 & \pm & 50.6 & $\mathrm{~ns}$ \\
TC & $(\mathrm{mg} / \mathrm{dL})$ & 186.1 & \pm & 13.6 & 201.3 & \pm & 13.9 & $\mathrm{~ns}$ \\
HDL-C & $(\mathrm{mg} / \mathrm{dL})$ & 63.5 & \pm & 4.8 & 68.1 & \pm & 4.2 & $\mathrm{~ns}$ \\
LDL-C & $(\mathrm{mg} / \mathrm{dL})$ & 108.8 & \pm & 10.8 & 118.7 & \pm & 11.3 & $\mathrm{~ns}$ \\
RemL-C & $(\mathrm{mg} / \mathrm{dL})$ & 6.2 & \pm & 0.7 & 7.5 & \pm & 1.1 & $\mathrm{~ns}$ \\
TG & $(\mathrm{mg} / \mathrm{dL})$ & 69.4 & \pm & 5.9 & 75.9 & \pm & 8.3 & $\mathrm{~ns}$ \\
RLP-TG & $(\mathrm{mg} / \mathrm{dL})$ & 11.0 & \pm & 1.0 & 12.5 & \pm & 1.2 & $\mathrm{~ns}$ \\
hTRL-TG & $(\mathrm{mg} / \mathrm{dL})$ & 61.3 & \pm & 6.4 & 70.3 & \pm & 9.0 & $\mathrm{~ns}$ \\
apoAI & $(\mathrm{mg} / \mathrm{dL})$ & 150.6 & \pm & 6.9 & 155.8 & \pm & 5.1 & $\mathrm{~ns}$ \\
apoAII & $(\mathrm{mg} / \mathrm{dL})$ & 24.7 & \pm & 0.5 & 26.1 & \pm & 0.3 & $\mathrm{~ns}$ \\
apoB & $(\mathrm{mg} / \mathrm{dL})$ & 79.3 & \pm & 7.2 & 83.6 & \pm & 7.8 & $\mathrm{~ns}$ \\
apoB48 & $(\mu \mathrm{g} / \mathrm{mL})$ & 3.5 & \pm & 0.4 & 3.7 & \pm & 0.6 & $\mathrm{~ns}$ \\
apoCII & $(\mathrm{mg} / \mathrm{dL})$ & 3.4 & \pm & 0.3 & 3.9 & \pm & 0.3 & $\mathrm{~ns}$ \\
apoCIII & $(\mathrm{mg} / \mathrm{dL})$ & 8.1 & \pm & 0.6 & 8.8 & \pm & 0.7 & $\mathrm{~ns}$ \\
apoE & $(\mathrm{mg} / \mathrm{dL})$ & 4.1 & \pm & 0.3 & 4.4 & \pm & 0.3 & $\mathrm{~ns}$ \\
\hline
\end{tabular}

ns: not significant

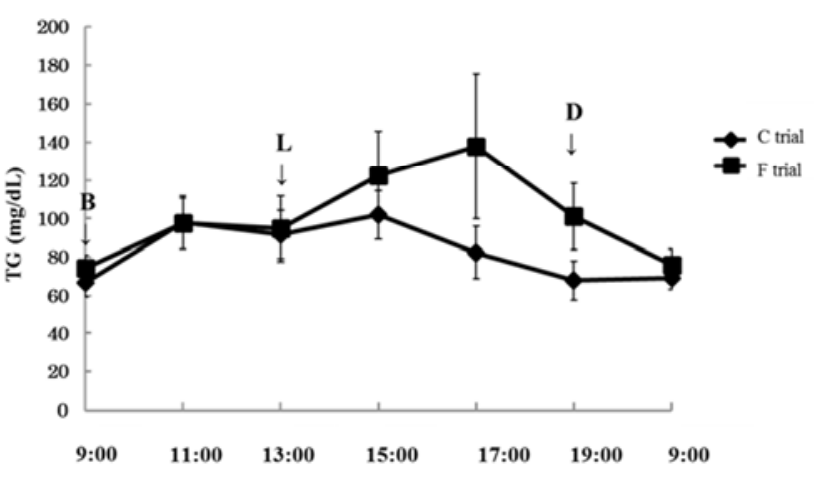

B: breakfast, L: lunch, D: dinner

Figure 1. Diurnal change of serum TG concentration. 


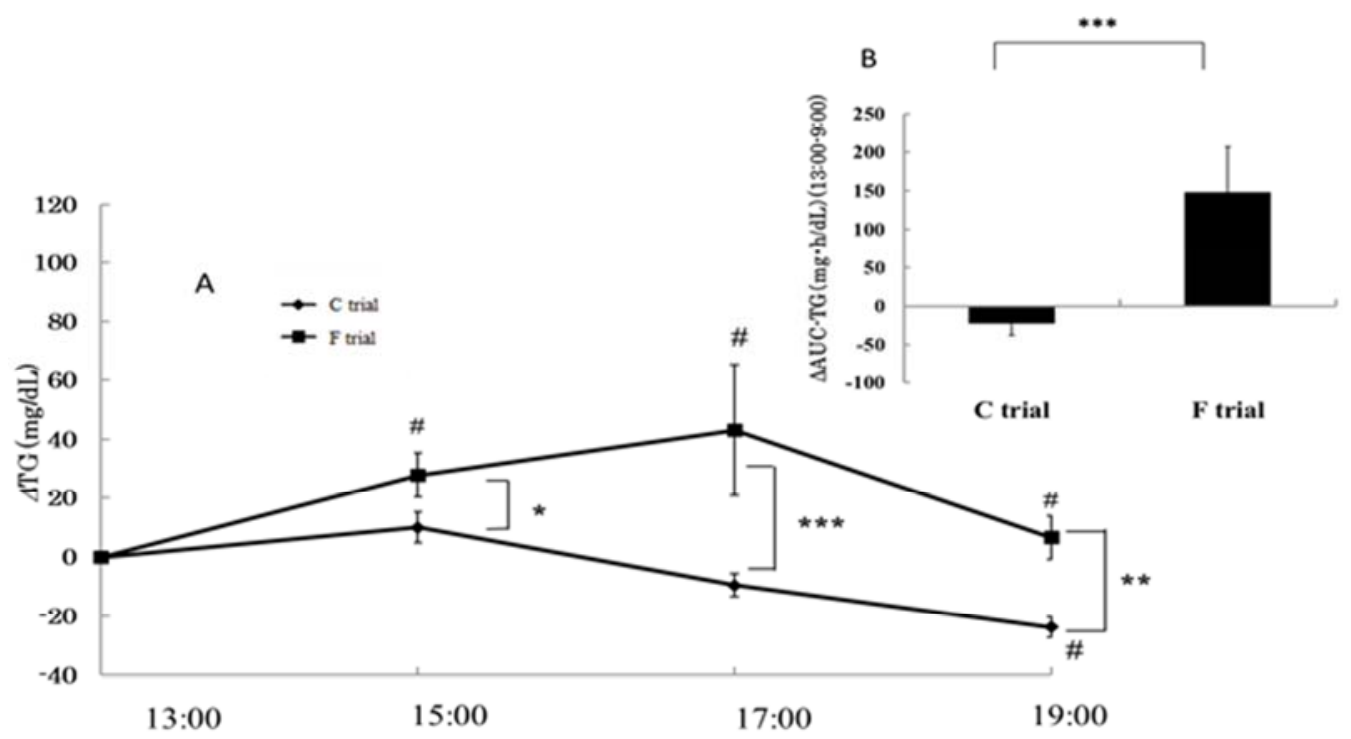

Figure 2. A: Diurnal change of $\triangle T G(13: 00-19: 00)$. B: $\triangle A U C-T G(13: 00-9: 00)$.

$\# p<0.05$ compared with baseline (13:00) in each trial.

${ }^{*} p<0.05,{ }^{* *} p<0.01, * * * p<0.001$ between the $\mathrm{C}$ trial and $\mathrm{F}$ trial.

Table 4. Pearson's correlation coefficients between $\triangle A U C-T G(13: 00-9: 00)$ and fasting serum TG, RLP-TG, hTRL-TG, RemL-C, apoB48, Glu, and insulin in the next morning.

\begin{tabular}{|c|c|c|c|c|c|c|c|c|}
\hline AAUC-TG (13:00-9:00) & & TG & RLP-TG & hTRL-TG & RemL-C & apoB48 & Glu & Insulin \\
\hline $\mathrm{C}$ trial & $r$ & 0.388 & 0.291 & $0.726^{*}$ & $0.742 *$ & 0.568 & -0.265 & 0.064 \\
\hline $\mathrm{F}$ trial & $r$ & -0.325 & $0.868 * *$ & 0.659 & $0.788^{*}$ & 0.398 & -0.169 & 0.015 \\
\hline
\end{tabular}

${ }^{*} p<0.05, * * p<0.01$

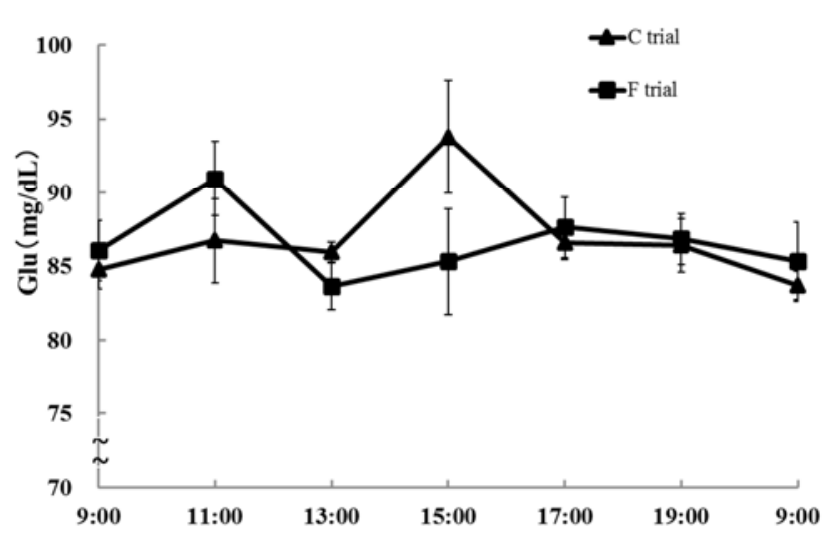

Figure 3. Diurnal change of serum Glu concentration.

\section{Discussion}

The major finding in this study is that after eating fast food (hamburger, fried potatoes, and cola) for lunch, serum TG concentration increased after $2 \mathrm{~h}$ and did not return to baseline even after $6 \mathrm{~h}$ (before dinner), although it did not increase after a lunch consisting of a common, non-fast-food diet was eaten. $\triangle \mathrm{AUC}-\mathrm{TG}$ after lunch until the evening was significantly larger after fast food was eaten compared to the non-fast-food lunch, although the effect did not last into the next morning. However, in the present study, because the subjects were healthy young women, it is important to point out the exaggerating effect of fast food ingestion on the postprandial TG response. These effects may be more serious for women after menopause or for subjects with conditions such as obesity, metabolic syndrome, and diabetes mellitus.

We previously studied the effects of menopause and age on postprandial lipoprotein responses after the ingestion of fat cream in healthy women and showed that: (1) the magnitude of postprandial TG concentration is dependent on age, but not on menopause, and (2) the clearance of remnant lipoproteins is delayed with age in pre- and post-menopausal women [13]. Our results [13] and those of others $[14,15]$ suggest a greater impact of aging on the pathways of TG than estrogen decline. Meanwhile, in healthy men, the diurnal TG profile is not age-dependent but is associated with insulin sensitivity, fat mass, and diet [16]. Waist circumference was the strongest determinant of postprandial lipidemia or diurnal TG profile in both males and females $[17,18]$. These results suggest that in postmenopausal women, the effect of eating fast food for lunch may be more harmful for their lipoprotein and lipid metabolism compared with young women. Further studies are warranted to elucidate the possible effect of eating fast food for lunch.

The fast food ingested in this study was typical fast food and was high in fat and energy-dense. Another feature is that the cola consumed contained a large amount of sugar, particularly fructose. In our previous studies, the postprandial TG, RLP-TG, and apoB48 concentrations following the coingestion of fat cream and fructose were significantly higher 
than those following the ingestion of fat cream alone or fat cream and glucose [5].

In addition, we also showed that the ingestion of fat cream with a high-fructose-syrup-containing beverage or the ingestion of a hamburger with cola (which contains a high amount of fructose) exaggerates and delays postprandial lipid and lipoprotein metabolism in young healthy women [6, 7]. In the present study, because the fast food ingested included fructose-containing cola, this may have influenced the postprandial lipid metabolism.

After common non-fast food was ingested at lunch, the serum TG concentration did not increase significantly, probably because the subjects were all healthy young women. In contrast, after fast food was ingested, the serum TG concentration increased, and this condition continued for more than $6 \mathrm{~h}$. In the present study, we did not adjust the energy of the food eaten at lunch between the common food and fast food because the aim of the study was to elucidate the effect of high-energy, high-fat fast food compared with common food. Really, the fast food was higher in energy, carbohydrate, and fat compared with the conventional lunch. However, it should be pointed out that the increase in serum TG concentration occurred in apparently healthy young women. Because postprandial lipidemia has been reported to be a significant and independent risk factor [1], in young people the daily ingestion of fast food may be deleterious for their health.

$\triangle \mathrm{AUC}-\mathrm{TG}$ from 13:00 to 9:00 the next morning correlated with the fasting concentrations of RemL-C and hTRL-TG. The methods for determining RemL-C and hTRL-TG may be more sensitive to endogenous remnants [19]. In contrast, $\triangle \mathrm{AUC}-\mathrm{TG}$ did not correlate with fasting apoB48 level in the next morning. These results indicate that the postprandial exogenous lipoprotein metabolism had ended before the next morning, but the endogenous lipoprotein metabolism had not finished even by the next morning. In the $\mathrm{F}$ trial, $\triangle \mathrm{AUC}-\mathrm{TG}$ correlated with the fasting RLP-TG level, which has been reported to be more sensitive to exogenous remnant [19]. This suggests that after the ingestion of fast food for lunch, the exogenous lipoprotein metabolism may not have finished even by the next morning. Dietary fat ingested at lunch has been reported to contribute to post-dinner lipidemia despite the relatively long interval between the 2 meals $(7 \mathrm{~h})$ [20]. Because high school pupils and university students have been reported to eat fast food mainly for lunch [8], the subjects took fast food as their lunch in the present study. However, fast food for dinner may be more deleterious because the TG level tends to increase from morning toward evening [15]. Further studies are needed to resolve these issues.

The serum glucose concentration showed no remarkable diurnal change from baseline (before breakfast), and there was no difference between the non-fast-food diet and the fast food diet, although the fast food contained more carbohydrate. This may be because the subjects who were enrolled in the present study were without glucose intolerance.

In summary, after fast food (hamburger, fried potatoes, and cola) was eaten at lunch, the serum TG level increased and did not return to baseline even after $6 \mathrm{~h}$, although it did not increase after a common non-fast-food lunch was eaten. The present results suggest that young women should not eat fast food frequently for lunch. However, because a relatively small number of subjects were examined in the present study, the results obtained should be interpreted with caution.

\section{Conclusion}

Compared with the conventional diet, the intake of fast food at lunch delayed the postprandial TG metabolism and the serum TG concentration did not return to baseline (before lunch) even before dinner in healthy young Japanese women. To eat fast food frequently for lunch may be deleterious for their health.

\section{Acknowledgments}

This study was supported by a Grant-in-Aid for Scientific Research (Basic Research C, JSPS KAKENHI Grant Number 24500874) from the Ministry of Education, Culture, Sports, Science and Technology of Japan. We would like to thank Mr. Ryuji Hayashi for his technical assistance. We also thank Ms. Yoshiko Achiwa and Ms. Airi Nakayama for their technical cooperation.

\section{References}

[1] Zilversmit DB. 1979. Atherogenesis: a postprandial phenomenon. Circulation 60: 473-485.

[2] Nordestgaard BG, Benn M, Schnohr P, Tybjaerg-Hansen A. 2007. Nonfasting triglycerides and risk of myocardial infarction, ischemic heart disease, and death in men and women. JAMA 298: 299-308.

[3] Bansal S, Buring JE, Rifai N, Mora S, Sacks FM, Ridker PM. 2007. Fasting compared with nonfasting triglycerides and risk of cardiovascular events in women. JAMA 298: 309-316.

[4] Tanaka A1, Tomie N, Nakano T, Nakajima K, Yui K, Tamura M, Numano F. 1998. Measurement of postprandial remnant-like particles (RLPs) following a fat-loading test. Clin Chim Acta 275: 43-52.

[5] Saito H, Kagaya M, Suzuki M, Yoshida A, Naito M. 2013. Simultaneous ingestion of fructose and fat exacerbates postprandial exogenous lipidemia in young healthy Japanese women. J Atheroscler Thromb 20: 591-600.

[6] Saito H, Kato M, Yoshida A, Naito M. 2015. The ingestion of a fructose-containing beverage combined with fat cream exacerbates postprandial lipidemia in young healthy women. J Atheroscler Thromb 22: 85-94.

[7] Saito H, Kato M, Yoshida A, Naito M. 2015. The ingestion of high-fructose syrup-containing cola with a hamburger delays postprandial lipid metabolism in young healthy Japanese women. J Food Nutr Sci 3: 139-146.

[8] Asano M, Fukakura N, Odachi J, Kawaraya C, Nanba A, Yasuda N, Yamamoto E. 2003. Use of fast foods among young people. Jpn J Nutr Diet 61:47-54 (In Japanese, Abstract in English). 
[9] Matthews DR, Hosker JP, Rudenski AS, Naylor BA, Treacher DF, Turner RC. 1985. Homeostasis model assessment: insulin resistance and beta-cell function from fasting plasma glucose and insulin concentrations in man. Diabetologia 28: 412-419.

[10] Okada M, Saito T, Yoshimura H, Noguchi Y, Ito T, Sasaki H, Hama H. 2005. Surfactant-based homogeneous assay for the measurement of triglyceride concentrations in VLDL and intermediate-density lipoprotein. Clin Chem 51: 1804-1810.

[11] Miyauchi K, Kayahara N, Ishigami M, Kuwata H, Mori H, Sugiuchi H, Irie T, Tanaka A, Yamashita S, Yamamura T. 2007. Development of a homogeneous assay to measure remnant lipoprotein cholesterol. Clin Chem 53: 2128-2135.

[12] National Health and Nutrition Survey, Ministry of Health, Labour and Welfare, Japan, 2011. http://www.mhlw.go.jp/bunya/kenkou/eiyou/h23-houkoku.htm 1

[13] Nabeno Y, Fukuchi Y, Matsutani Y, Naito M. 2007. Influence of aging and menopause on postprandial lipoprotein responses in healthy adult women. J Atheroscler Thromb 14: 142-150.

[14] Castro Cabezas M, Halkes CJ, Meijssen S, van Oostrom AJ, Erkelens DW. 2001. Diurnal triglyceride profiles: a novel approach to study triglyceride changes. Atherosclerosis 155: 219-228.

[15] Jackson KG, Abraham EC, Smith AM, Murray P, O'Malley B, Williams CM, Minihane AM. 2010. Impact of age and menopausal status on the postprandial triacylglycerol response in healthy women. Atherosclerosis 208: 246-252.

[16] van Oostrom AJ, Castro Cabezas M, Ribalta J, Masana L, Twickler TB, Remijnse TA, Erkelens DW. 2000. Diurnal triglyceride profiles in healthy normolipidemic male subjects are associated to insulin sensitivity, body composition and diet. Eur J Clin Invest 30: 964-971.

[17] Nabeno-Kaeriyama Y, Fukuchi Y, Hayashi S, Kimura T, Tanaka A, Naito M. 2010. Delayed postprandial metabolism of triglyceride-rich lipoproteins in obese young men compared to lean young men. Clin Chim Acta 411:1694-1699.

[18] Halkes CJ, Castro Cabezas M, van Wijk JP, Erkelens DW. 2001 Gender differences in diurnal triglyceridemia in lean and overweight subjects. Int $\mathrm{J}$ Obes Relat Metab Disord 25: 1767-1774.

[19] Sato I, Ishikawa Y, Ishimoto A, Katsura S, Toyokawa A, Hayashi F, Kawano S, Fujioka Y, Yamashita S, Kumagai S. 2009. Significance of measuring serum concentrations of remnant lipoproteins and apolipoprotein B-48 in fasting period. J Atheroscler Thromb 16: 12-20.

[20] Maillot F, Garrigue MA, Pinault M, Objois M, Théret V, Lamisse F, Hoinard C, Antoine JM, Lairon D, Couet C. 2005. Changes in plasma triacylglycerol concentrations after sequential lunch and dinner in healthy subjects. Diabetes Metab 31: 69-77. 